SHS Web of Conferences 7, 01006 (2014)

DOI: $10.1051 /$ shsconf / 20140701006

C) Owned by the authors, published by EDP Sciences, 2014

\title{
Analysis of Regional Economic Disparities in Guizhou Province Based on ESDA-GIS
}

\author{
Wang Haili, Yuan Tianfeng, Hu Xiaodong, Qu Xiaobin \\ School of Geography \& Resource Science, Neijiang Normal University, 641100 Neijiang, China
}

\begin{abstract}
Take the county as the research scale and the per capita GDP as the measure index as well to reveal the difference of Guizhou Province's regional economy which based on ESDA and GeoDA-GIS. It shows that the level of economic develop of Guizhou's central area is high and surrounded area is low. The difference between North and south is greater than the difference between East and West. There is a clear spatial correlation among them. Moran scatter diagram shows that the majority of counties are located in the first and third quadrants, which accounted for $73.86 \%$ of the total number of the county. The number of "L-L" type is more than the number of "H-H" type 37 counties. Most parts of the provinces are relatively poor. Finding the "H-H" area and "L-L" area and "L-H" area and "H-L" area of economic development level of county based on the spatial correlation model. That can provide scientific basis for the future economic construction and social development of Guizhou province.
\end{abstract}

Keywords. regional economic disparity; spatial autocorrelation; aggregation

\section{Introduction}

Regional economic disparity refers to inequality of general economic development level in terms of per capita among national regions in a certain period[1]. It is a common phenomenon in the regional economic development course, and its existence and variation will directly and indirectly affect many aspects like regional economy and social development, which make it one of the focuses in the economic and social life[2]. Currently, the research findings from scholars of regional economic disparity mainly focus on analysis of current situation and spacial-temporal evolution[3-4] of regional economic disparity, as well as the coordination development [5-6] of regional economy. The research objects concentrate more on the provinces and regions with relatively developed economy, and less on the regional economic disparity of western depressed areas[8-9]; In terms of the research methods, it frequently adopts standard deviation, Theil index, Gini coefficient, principal component-cluster analysis[10-13], and there are less research findings obtained from using combination of exploratory spatial data analysis (ESDA) and GeoDA-GIS platform[8,9,14]; Partial researches of regional economic disparity in Guizhou Province have been conducted by scholars[15-20], but due to the limitation of data, techniques and talents, there's no relevant finding obtained from researches of regional economic disparity in Guizhou using ESDA technique and GeoDA-GIS platform system in spatial perspective by any scholars. Based on the great number of researches which have been conducted on regional economic disparity by scholars, this article takes Guizhou Province as its research object, county as its research unit, and uses ESDA technique and GeoDA-GIS platform to 
make a research on regional economic disparity of Guizhou Province in 2011, for the purpose of revealing regional economic disparity in Guizhou Province, expecting to provide a scientific basis for the future economic construction and social development planning of Guizhou Province.

\section{Research region}

Located in the southwest of Chinese inland, Guizhou Province lies between east longitude of $103^{\circ} 33^{\prime}$ $109^{\circ} 35^{\prime}$ and north latitude of $24^{\circ} 37^{\prime}-29^{\circ} 13^{\prime}$. It faces Hunan in the east, Guangxi in the south, Yunnan in the west and Sichuan and Chongqin in the north, with an area of about 176 thousand square kilometers. Its inner terrain is high in the west and low in the east, with extensive coverage of Custer landform, larger area of mountains and highlands and smaller area of plains. The whole province is located in moist monsoon climate region of subtropics with abundant rainfall and rich river systems, and is the watershed of pearl river and Yangtze river basin; It contains plenty of mineral resources, which makes it a significant resource province and industrial base in the southwest region. In 2011, the gross regional product hit 570.2 billion yuan, ranking the last among three provinces and one city in the southwest of China, but its per capita GDP of about 16,436.55 yuan ranked the last in China, which made it a province with extremely backward economic development level in China. The highest provincial per capita GDP of 16436.55 yuan in Renhuai City is 7.61 times more than the lowest provincial per capita GDP of 6196.72 yuan in Wangmo County, which demonstrates a very obvious regional disparity of economic development level.

\section{Research method and data source}

\subsection{Research method}

\subsubsection{Spatial weight matrix}

To reveal the spatial connections among phenomena, the measurement may be conducted by defining spatial weight matrix. The most common rules for determination of spatial weight matrix include simple binary adjacency matrix and binary spatial weight matrix which is based on distance. This article adopts the spatial connections that are based on adjacency to construct the weight matrix, as shown in the following:

$$
W_{i j}= \begin{cases}1 & \text { Region } i \text { is adjacent to region } j \\ 0 & \text { Region } i \text { is not adjacent to region } j\end{cases}
$$

\subsubsection{Analysis of global autocorrelation}

Global autocorrelation describes the spatial concentration feature and strength in the whole region for a certain phenomenon, and its determining indexes generally include global indexes of Moran index and Geary index, in which the Moran index is used more frequently. This article adopts Moran index. If $x_{i}$ is an observed value in a certain region, the global Moran index I of this regional variable shall use the following formula[22]:

$$
I=\frac{n \sum_{i=1}^{n} \sum_{j \neq 1}^{n} w i j\left(x_{i} \quad \bar{x}\right)\left(x_{j} \quad \bar{x}\right)}{S^{2} \sum_{i=1}^{n} \sum_{j \neq 1}^{n} w_{i j}}
$$


In this formula: I is Moran index; $S^{2}=\frac{1}{n} \sum_{i=1}^{n}\left(x_{i}-\bar{x}\right) ; \bar{x}=\frac{1}{n} \sum_{i=1}^{n} x_{i}$

Moran index I ranges from -1 and 1 , and the value lower than 0 indicates negative correlation, equaling 0 indicating non-correlation and higher than 0 indicating positive correlation. The significance test of Moran index adopts $\mathrm{z}$ test.

\subsubsection{Analysis of local autocorrelation}

Analysis of global autocorrelation is the global analysis of spatial autocorrelation, which ignores the local conditions. To further reveal the concentration feature of local space, the local index of spatial connection Local Moran's I index shall be adopted, and its calculation formula is as follows[22]:

$$
I_{i}=\frac{x_{i} \quad \bar{x}}{S^{2}} \sum_{j \neq i}^{n} w_{i j}\left(x_{j} \quad \bar{x}\right)
$$

In which, $n 、 x_{i} 、 x_{j} 、 w_{i j} 、 S^{2} 、 \bar{x}$ are the same as those in global autocorrelation formula, and the meaning of Ii is similar to global index. The significance test of Moran's I index is similar to global index test.

\subsection{Data source}

The regional per capita GDP data adopted by this article is from the edited Statistical Yearbook of Guizhou Province 2012, and it has a high credibility and comprehensiveness. The vector graph of county-level administrative division adopts scanned and vectorized drawing of regional administrative division in 2011, which contains totally 88 county-level administrative units.

\section{Regional economic and spatial disparity}

\subsection{General feature of spatial disparity}

\subsubsection{Trend of spatial disparity}

Trends Analysis tool provides users with sampling points in research area to be converted to 3D perspective taking the interested attribute values as the height. It allows users to analyze the global trend of sampled data collection in difference visual angles[23]. In the trend analysis perspective, every vertical bar represents the value (height) and location of a data point. These points are projected to two orthogonal planes, one in east-west direction and the other in south-north direction. A fitted line can be produced by the project points and then used to simulate the trend in a certain direction[23]. In the ArcGIS platform, the Trends Analysis tool will be called to dynamically observe the regional per capital GDP distribution trend in the whole province, and then draw a 3D perspective (as in Figure 1). The result indicates that: if seen from the east-west direction, the middle part is higher than the west part, while the west part is little higher than the east part, but if seen from the south-north direction, the middle part is higher than the south-north part, and it generally displays a trend in which the middle part is high and its surrounding part is low. 


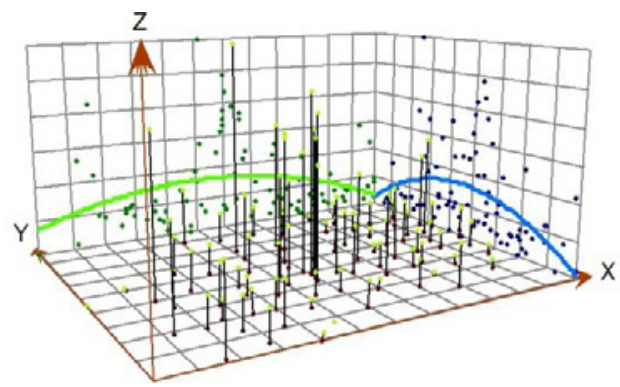

Figure 1. 3D perspective for spatial trend of regional economy

\subsubsection{Spatial concentration feature}

Global spatial autocorrelation analysis of regional per capital GDP in Guizhou Province is made by calling spatial Autocorrelation (Global Moran's I) tool in ArcGIS10 and adopting Polygon contiguity (First Order) as the judgment principle of spatial connection. The Moran's I is 0.407, Z 6.585, standardized $\mathrm{Z}$ value 6.585 , which are higher than bilateral test threshold of 2.58 for normal distribution in the $99 \%$ of confidence interval. So the test is passed. The result indicates that the regional per capital GDP in Guizhou Province possesses a very conspicuous spatial concentration feature, and the counties with relatively high per capita GDP are adjacent to those with relatively high per capita GDP, while the counties with relatively low per capita GDP are adjacent to those with relatively high low capita GDP, from which it can be inferred that regional polarization is a significant feature of economic development in Guizhou Province.

\subsection{Local spatial disparity feature}

\subsubsection{Analysis of Moran scattered diagram}

Analysis of global autocorrelation can only reflect the concentration degree of phenomenon in allaround space, but cannot determine the detailed region. While the Moran scattered diagram can be used to research the local spatial disparity feature. Use GeoDA software to draw a Moran scattered diagram (as in Figure 2), and draw a corresponding Moran concentrated diagram in ArcGIS (as in Figure 3) to directly show the concentration feature of local space. In Figure 2, the amount of counties in the first and third quadrant is obviously higher than that in the second and fourth quadrant, which indicates that the regional economic development level of Guizhou Province spatially contains a obvious dualistic structure. Through statistics, there are 14 counties in the first quadrant, accounting for $15.909 \%$ of total amount of regions. While there are 51 counties in the third quadrant, accounting for $57.955 \%$ of total amount of regions. The combination of them accounts for $73.864 \%$ of total amount of regions, and there are 37 more counties of "low-low" concentration than those of "highhigh" concentration, which indicates that the economical development level is low for most of regions in Guizhou Province, while the counties with relatively high economic development level occupy a little area only. The regional areas of "low-low" type are extensively distributed in the eastern, southern and western regions of Guizhou Province. The main reason is that Guizhou has a Custer landform and rugged topography, and the living and production condition of people is commonly poor, together with a low level of economic development which leads the whole province to a common poverty. However, the altitude of Guiyang basin is moderate, with relatively flat topography. So the natural condition is superior to that in other places. The remaining 23 counties is scattered in above two types. The "L-H" type in the second quadrant is mainly distributed around the "H-H" type, while the "H-L"-type of counties in the fourth quadrant is scattered in "L-L" type or the edge of provincial boundaries in island shape. The reason is that the economic development level is low for most of regions in Guizhou Province, and the natural condition/ resource basis, traffic location, etc. of some 
counties are relatively good, with the economic development level obviously higher than that in its surrounding regions.

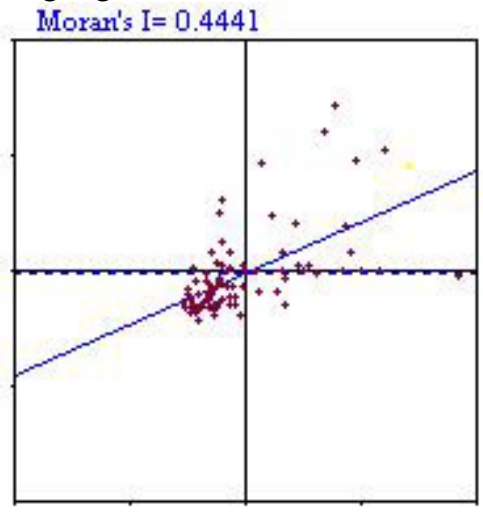

Figure 2. Moran scattered diagram

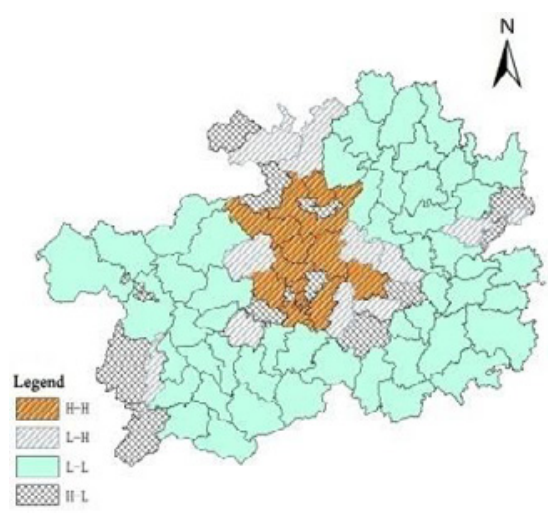

Figure 3. Moran concentrated diagram

\subsubsection{Analysis of LISA concentrated diagram}

The analysis of Moran scattered diagram helps to preliminarily understand the regional economic disparity feature of the whole province and find relatively developed and backward regions. To further measure the spatial autocorrelation of regional economy in Guizhou Province and to find the hot and blind spot of regional economic development, LISA concentrated diagram is produced in GeoDA software. (as in Figure 3).

The regions with relatively high spatial concentration of economic development level in Guizhou Province are mainly distributed in Guiyang basin of middle part and borderlands of southeast part in Guizhou Province. Guiyang basin in the middle part belongs to central economic zone of Guizhou Province, and its topography is relatively flat. The location of provincial capital Guiyang is the political, economic and cultural center, as well as the traffic hub. The relations among each region is close, and most of the regions present "H-H" concentration, belonging to high-high raised areas, which means the counties with relatively high economic development level are surrounded by the counties with relatively high economic development level, and it is the hot spot for economic development.

The eastern borderland is mainly the distribution areas of counties with relatively low economic development level, presenting "L-L" concentration and belonging to low-low collapsed area, which means the counties with relatively low economic development level are surrounded by the counties with relatively low economic development level, and it is the cold spot for economic development. The main reason is that in this region, the topography is rugged, with scarce resources, backward traffic infrastructure; it is distant from central city, and the economic development level of bordered areas in every direction is not high, therefore this region is difficult to be affected by the radiation of regions with developed economy.

Most of the regions distributed around the "H-H" type present "L-H" concentration, belonging to low-high hollow area, which means the economic development level in the central counties is relatively low but it's high in the surrounding regions. This type of area shall take full advantage of its location in the surrounding regions with developed economy to gain the economic development experience to promote its own development and endeavor to reduce the gap with surrounding regions. Bijiang Area, Yuping County and Pan County embedded in "L-L" concentration type and borderland of unobvious-type counties present "H-L" concentration, belonging to high-low island-shaped area, which means the economic development level in the central counties is relatively high but it's low in the surrounding regions. The main reason is that those three counties have good traffic conditions, abundant mineral resources, strong industrial basis, fast economic development and high economic development level, which make them extremely different from their surrounding regions with 
relatively low economic development level. It presents an obvious "H-L" concentration feature, displaying a state of isolated island-shaped distribution.

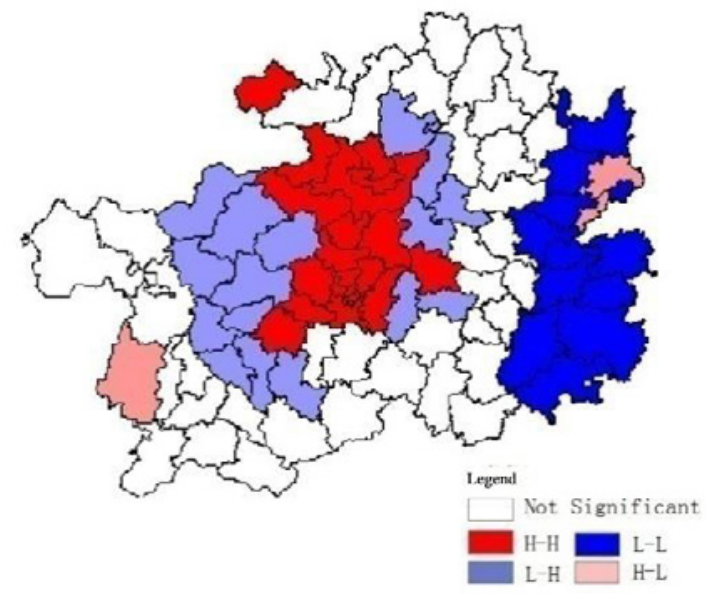

Figure 4. LISA concentrated diagram

Furthermore, the spatial correlation among many regions is not that obvious. The economic development level of regions in semiring shape and distributed in the west, south, east and northeast part of Guizhou presents a relatively strong heterogeneity inside and outside the regions.

\section{Conclusions}

The economic disparity feature of Guizhou regions is revealed through the above analysis, and it can be concluded that: (1) Trends analysis indicates that in Guizhou Province, the regional economic development level in the middle part is high and the same in its surrounding part is low; (2) The analysis of global spatial autocorrelation indicates that the regional economy of Guizhou Province has an extremely strong spatial autocorrelation and obvious spatial concentration feature; (3) Local spatial correlation reveals a heterogeneity feature of local space for regional economy in the whole province, and finds that most of the regions in the whole province are poor, and a spatial dualistic structure for a small part of counties with relatively high economic development level, as well as the hot and cold spots for economic development is discovered.

Based on the regional spatial disparity feature in Guizhou Province, the advice is provided as follows: (1) Completely understand the objective fact that the economic poverty is all over Guizhou Province and the regional disparity is very conspicuous, make great efforts to develop the economic growth pole in the middle part of Guizhou and growth center in regions such as Liupanshui, zunyi, etc., break the regional chain of common poverty and increase its radiation-driven function on regional economic growth; (2) Strengthen the poverty alleviation for poor regions, expand poverty alleviation channel and perfect poverty alleviation system to strive for eliminating poverty and facilitate the coordinated development of regional economy; (3) Accelerate the comprehensive traffic planning and construction of the whole province, solve difficult-access problem and facilitate the free flow of economic factors in and out of regions; (4) Especially further invest the education in minority regions to improve their cognitive level and labor skills, strengthen their employment and selfdeveloping ability and completely eliminate the gene of poor generation. 


\section{Acknowledgement}

This paper is supported by Scientific Research Project of Neijiang Normal University (GN:13ZB10); "Comprehensive Reform of Geographical Science Major" of Higher Education Quality Engineering Project of Neijiang Normal University 2011(GN:P01256).

\section{References}

1. Tan Chenglin. Research on Chinese Regional Economic Disparity[J]. Beijing: China Economic Publishing House, 1997:35-38.

2. Li Xiaojian, Li Guoping, Zeng Gang, etc.. Economic Geography[J]. Beijing: Higher Education Press, 2006:247.

3. Ou Xiangjun, Shen zhengping, Zhu Chuangeng. Spatial Analysis of Regional Economic Disparity Evolution in Jiangsu Province [J]. Economic Geography, 2007, 27(1):78-83.

4. Hao Junqing, Wang Yanlin, Yao Hong, etc..Spatial Analysis of Regional Economic Disparity Evolution in Yanan City [J]. Resources and Environment in Arid Region, 2008, 22(8):12-16.

5. Shang Xiaomei, Zhou Shituan, Yin Zhencai. Analysis of Regional Economic Disparity and Research of its Coordinated Development Strategy in Hebei Province[J]. Journal of Hebei University of Science and Technology (Social science edition), 2012, 12(6):34-41.

6. Yin Laisheng, Feng Bangyan, Li Shenghui. Regional Development Disparity and Spatial Framework Evolution in Guangdong [J].Geographic Research and Development, 2012, 31(1):3034.

7. Guan Wei, Liu Yongfeng. Spatial-Temporal Evolution of Coordinated Development Degree of Liaoning Coastal Economic Zone and Environment [J]. Geographic Research, 2012, 31(11):20452054

8. Zhang Haifeng, Bai Yongping, Chen Qiong, etc..Research of Regional Economic Disparity in Qinghai Province Based on ESDA-GIS [J]. Geography in Arid Region, 2009, 32(3):454-460.

9. Pan Jinghu, Feng Zhaodong, Dong Xiaofeng. ESDA-GIS of Spatial-temporal Framework of Regional Economy in Gansu Province [J]. Journal of Lanzhou University (Natural science edition), 2008, 44(4):45-50.

10. Sun Xihua, Zhang Shumin. Analysis of Regional Economic Disparity and Research of its Coordinated Development in Shandong Province[J]. Economic Geography, 2003, 23(5):611-620.

11. $\mathrm{Yu}$ Hanzheng, $\mathrm{Xu}$ Chenglong. Research on the Trend of Regional Economic Disparity in Shandong Province. Resource Development and Market[J], 2012, 28(1):48-49.

12. Li Li, Guan Weihua. Research of Regional Economic Disparity Framework since Reform and Opening up[J]. Economic Geography, 2010, 30(10):1605-1611.

13. Wu Leying, Miao Changhong. Research of Regional Economic Disparity and its Coordinated Development in Henan Province[J]. Henan Science. 2012, 30(3):372-375.

14. Zeng Yongming, Zhang Guo. Research of Regional Economic Disparity in Sichuan Province Based on GeoDA-GIS[J]. Research of Yunnan Geographical Environment, 2010, 22(4):55-58.

15. Liu Qun, Wang Yingzhe. Comparative Analysis of Social Development Level of Regional Economy in Guizhou [J]. Economic Geography (Supplementary issue), 2006(26):53-55.

16. Long Yongjun, Zhang Daoyi. Analysis of County-level Regional Economic Disparity in Guizhou Province[J]. Journal of Guizhou Normal College (Natural Science), 2007, 18(2):63-67.

17. Zhang Lei, Li Weitao, Wang Qiulong. Measurement and Evaluation of Regional Economic Development Disparity in Guizhou in 2000-2007[J]. Journal of Guizhou Normal College (Natural Science), 2008, 19(6):73-76.

18. Chen Qunli, Wang Hong. Research of Spatial Disparity Framework of Regional Economic Disparity in Guizhou Province[J]. Research of Territory and Natural Resources, 2011(3):21-22.

19. Jiang Qingqiong, Tian Yingfu. Covariance Analysis of Regional Economic Disparity in Guizhou Province [J]. Mathematical Statistics and Management, 2012, 31(4):613-620.

20. Zhu Shipeng, Kuang Xuedong, Zhang Meizhu. Measuring Analysis of Regional Economic Disparity in Guizhou Province [J]. Journal of Xibei Normal University (Natural science edition), 2012, 48(4):102-107. 
21. Xu Jianhua. Geographic Modeling Method[M]. Beijing: Science Press, 2010, 85.

22. Lv Chen, Fan Jie, Sun Wei. Research of Spatial Framework for Chinese Population and its Influencing Factors Based on ESDA [J]. Economic Geography, 2009(11):1797-1802.

23. Tang Guoan, Yang Xin. ArcGIS Experimental Instruction of Spatial Analysis on Geographic Information System [M]. Beijing: Science Press, 2006:378-379. 